\title{
Assessment of In Vivo Lumbar Inter-Vertebral Motion: Reliability of a Novel Dynamic Weight- Bearing Magnetic Resonance Imaging Technique Using a Side-Bending Task
}

\author{
Niladri Kumar Mahato ${ }^{1,2,3}$, Stephane Montuelle ${ }^{3}$, Brian C. Clark ${ }^{2,3,4}$ \\ ${ }^{1}$ The University of The West Indies, St. Augustine, Trinidad and Tobago \\ ${ }^{2}$ Ohio Musculoskeletal and Neurological Institute, Ohio University, Athens, OH, USA \\ ${ }^{3}$ Department of Biomedical Sciences, Ohio University, Athens, OH, USA \\ ${ }^{4}$ Department of Geriatric Medicine, Ohio University, Athens, OH, USA
}

Study Design: Between-session reliability of a magnetic resonance imaging (MRI) based experimental technique to quantify lumbar inter-vertebral motion in humans.

Purpose: We have developed a novel, dynamic, MRI-based approach for quantifying in vivo lumbar inter-vertebral motion. In this study, we present the protocol's reliability results to quantify inter-vertebral spine motion.

Overview of Literature: Morphometric studies on intervertebral displacements using static, supine MRI and quantification of dynamic spine motion using different $X$-ray based radiography techniques are commonly found in the literature. However, reliability testing of techniques assessing real-time lumbar intervertebral motion using weight-bearing MRI has rarely been reported.

Methods: Ten adults without a history of back pain performed a side-bending task on two separate occasions, inside an open-MRI, in a weight-bearing, upright position. The images were acquired during the task using a dynamic magnetic resonance (MR) sequence. The MRI imaging space was externally calibrated before the study to recreate the imaging volume for subsequent use in an animation software. The dynamic MR images were processed to create side-bending movement animations in the virtual environment. Participant-specific three-dimensional models were manually superimposed over vertebral image silhouettes in a sequence of image frames, representing the motion trials. Inter-vertebral axes and translation and rotational displacements of vertebrae were quantified using the animation software.

Results: Quantification of inter-vertebral rotations and translations shows high reliability. Between-session reliability results yielded high values for the intra-class correlation coefficient $(0.86-0.93)$, coefficient of variation $(13.3 \%-16.04 \%)$, and Pearson's correlation coefficients (0.89-0.98)

Conclusions: This technique may be developed further to improve its speed and accuracy for diagnostic applications, to study in vivo spine stability, and to assess outcomes of surgical and non-surgical interventions applied to manage pathological spine motion.

Keywords: Magnetic resonance imaging; Lumbar vertebrae; Intervertebral disc; Recurrent low back pain

Received Aug 27, 2018; Revised Oct 5, 2018; Accepted Oct 6, 2018

Corresponding author: Niladri Kumar Mahato

Department of Preclinical Sciences, Faculty of Medical Sciences, The University of The West Indies, St. Augustine Campus, Trinidad and Tobago

Department of Biomedical Sciences, Ohio Musculoskeletal and Neurological Institute, Ohio University, Athens, 45701, OH, USA

Tel: +1-868-372-6894, Fax: +1-868-662-9148, E-mail: Niladri.Mahato@sta.uwi.edu,nm620511@ohio.edu 


\section{Introduction}

Spine motion assessment is performed during patient examination if restricted movement is suspected to involve spine disorders, hyper-mobility at a vertebral joint is linked to recurrent low back pain, or if evaluation of outcomes in surgical and non-surgical interventions on the spine is necessary [1-5]. Common spine motion assessment methods use palpatory techniques on surface landmarks, which often underrepresent actual spine motion $[6,7]$. Even newer, non-invasive techniques using sensors placed superficially on the skin are less than ideal for kinematic assessment of spine or other joint motion due to errors caused by soft-tissue movement over the vertebrae or bony condyles [7-9].

In clinical practice, using magnetic resonance imaging (MRI) for objective assessment of spine disorder or low back pain (LBP) has mostly been limited to evaluation of static images in the supine position. More recently, instrumented spine loading and 'positional' spine scanning have been performed in the sitting, weight-bearing while supine, or standing positions [10-13]. However, weightbearing MRI yields static images and may take several minutes to acquire using routine sequences. For the same reason, acquisition of 'positional', end-of-range magnetic resonance (MR) images may be challenging in subjects with concurrent back pain. However, several assortments of fast, gradient based MRI sequences have been developed more recently to acquire rapid, real-time spine motion images [14,15].

We recently reported a fast MRI (True-FISP [fast imaging with steady-state precession] like) streaming sequence technique, using the two-dimensional (2D)-hybrid contrast enhanced streaming (HyceS) that can reliably measure pre-determined displacements in custom-built, solidbody objects and porcine spine models within a calibrated MR imaging volume $[16,17]$. In this study, we sought to translate our work to humans and determine the technique's reliability in quantifying lumbar vertebral motion. We used an ultra-fast streaming sequence to scan the lumbar spine in real-time while study participants performed a lateral-bending task in weight-bearing position (standing), in an open magnet system, on two different days. Using image based three-dimensional (3D) models of individual vertebrae, and manual matching (rotoscoping) onto corresponding vertebral silhouettes from coronal image slices, dynamic inter-vertebral spine displacements were measured during the lateral-bending trials [18]. This study presents the reliability results from this novel dynamic MRI research protocol we have developed to quantify dynamic inter-vertebral spine displacements.

1. Calibrating magnetic resonance imaging volume Constructing grid Scanning and segmenting grid Calibrating lumbar coil volume

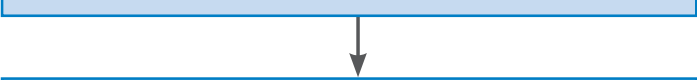

2. Creating animation environment Setting-up Maya cameras

3. Explaining the Imaging protocol Static lumbar spine scanning Acquisition of dynamic motion trials

4. Image processing Importing trial images and three-dimensional models

(A) Rotoscoping, animating, and quantifying in virtual envi ronment

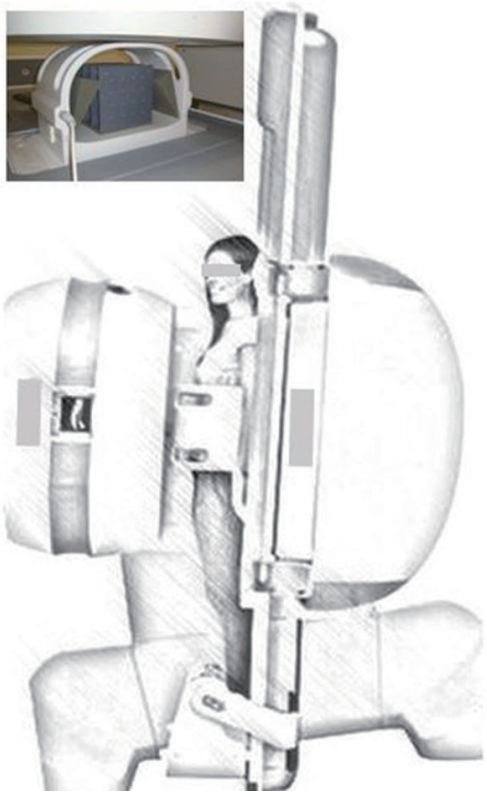

Fig. 1. (A) Overall design of the technique presented as four sequential steps. (B) Weight-bearing position of the magnetic resonance imaging scanner (tilted from the supine to an upright position, approximately $87^{\circ}$ to the horizontal). A subject is standing upright with her lumbar region surrounded by a lumbar coil. Inset (top left) shows calibration grid inside the lumbar coil used to calibrate the coil. 


\section{Materials and Methods}

\section{General overview of the technique}

This technique can be split into four major steps, as outlined below. An open, weight-bearing MRI system (0.25 T; Esaote G-scan Brio, Genoa, Italy) was used for imaging in this study. This MR imaging system's gantry allowed for tilt from the horizontal to the vertical orientation in small increments. This facility allows us to acquire images in a weight-bearing position (Fig. 1). The main magnet in this machine is 'open' on the left side of the subject, alleviating claustrophobia within the imaging environment while simultaneously allowing enough space for the sidebending protocol designed for this study. Step 1 involved a one-time calibration of the MR imaging volume within the MRI system's lumbar coil using a custom-built grid system before data acquisition (Fig. 1, inset). Step 2 involved creating the virtual animation environment using the previously acquired volume calibration data. Step 3 involved imaging the study volunteers. Stand-alone axial images of the L2-L4 vertebrae were acquired for segmentation (AVIZO software, Hillsboro, OR, USA). A timed, side-bending protocol was followed to perform the motion trials. An ultra-fast, 2D-HyceS dynamic imaging sequence was used to acquire images during the motion trials. Motion trials were imaged in two separate sessions. Step 4 involved post-processing the Digital Imaging and Communications in Medicine (DICOM) images acquired from the motion trials. The animation software AutoDesk MAYA (Autodesk Inc., San Rafael, CA, USA) was used to deconstruct the dynamic trial images into image frame sequences. Three-D models of motion segments were generated and imported into the MAYA animation program. These 3D models were then manually superimposed (using scientific rotoscoping) onto their corresponding outlines/silhouettes across all trial image frames [18]. The animation software calculated movements of inter-vertebral axis motion as well as motion in each vertebra within the imaging volume, taking the neutral, erect position as the reference (zero displacement) point. The details of creating the motion animation in the MAYA software (Autodesk Inc.), such as creating MAYA framespecs files, digitizing the calibration beads, scientific rotoscoping, and animation and quantification protocols, are described in previous work $[17,19]$.

\section{Participant selection}

Twelve volunteers between 18 and 60 years of age participated in this study. Individuals were excluded if they reported LBP $\geq 2$ on the Numeric Pain Rating (NPR) Scale at the screening. Subjects with a history of spinal surgery, orthopedic or neurologic impairments, cancer or tumors, cardiopulmonary disorders, and/or clinical depression were excluded, as were individuals taking medications or supplements for LBP. Lastly, study participants were screened for general compatibility for MRI scanning, and subjects with a body mass index (BMI) greater than $32 \mathrm{~kg} /$ $\mathrm{m}^{2}$ or with any debilitating condition that impaired voluntary spine-bending movement were also excluded from the study for safety reasons and for the convenience of executing the trunk bending task (in weight-bearing position) inside the limited space available within the MRI system's lumbar coil. All subjects gave written, informed consent. This study was approved by the Ohio University Institutional Review Board (OU IRB \#16-F-13). The study required three visits to the laboratory. Visit 1 was used for subject consent and orientation to the study. The participants became familiar with the MR imaging environment, practicing the simulated and timed motion trials they would perform on their subsequent experimental visits. Visits 2 and 3 were scheduled within a week of each other at the MRI facility for dynamic imaging. Out of the 12 participants screened, 10 completed the study protocol. The investigators withdrew one participant due to concerns over limitations of performing the bending task inside the MR lumbar coil (i.e., while they were within the established BMI limits, there was insufficient space for the subject to perform the task), and the other was excluded due to reporting LBP $>2$ on the NPR Scale at screening.

\section{Protocol for motion trial imaging}

Supine, axial T2 weighted images of L2-L4 segments were acquired (approximately 9 minutes) using a fast spinecho sequence for 3D modeling of the lumbar vertebrae (repetition time [TR], 6,969 ms; echo time [TE], $100 \mathrm{~ms}$; number of excitations [NEX], 1; matrix, $224 \times 220 \mathrm{~mm}$; oversampling, $155 \%$; field of view [FOV], $240 \times 240 \mathrm{~mm}$; no gap). With the height of the foot-rest adjusted and recorded, the table was rotated to the $87^{\circ}$ vertical as the participant eased into the new weight-bearing position. The subject's pelvis was tightly strapped across the hip 
joints with a safety belt to minimize pelvic motion during trials. The participants were asked to bend laterally to their right, sliding their hands to the sides of their thighs as far as they could (maximum excursion was noted and a vitamin E pill was taped to a thigh strap Velcroed around the subject's clothing, as a reference target for the actual trials) or till their bending was physically restricted by the system's surrounding lumbar coil, whichever occurred first.

During the trials, the subjects began in an erect (neutral) position and bent laterally at the lumbar spine (without tilting the pelvis). The task was performed over a 20 -second duration (i.e., 10 seconds to bend to the side and 10 seconds to come back to neutral). The participants received audio feedback of verbal counts from a metronome through an MRI compatible headphone system. Five repetitions per trial were performed. Three trials were completed with a 30 -second rest between trials. Right-sided bending was chosen for our imaging protocol due to the restricted space available within the lumbar coil for performing flexion-extension motion or left-sided bending within the coil (i.e., the signal cord connector to the main magnet was on the left side of the coil and would have interfered with moving in the magnet).

A 2D-HyceS sequence was used for imaging to acquire continuous, ultra-fast coronal lumbar spine imaging (TR, $7 \mathrm{~ms}$; TE, $3.5 \mathrm{~ms}$; NEX, 1; matrix, $180 \times 180 \mathrm{~mm}$; FOV, $320 \times 320 \mathrm{~mm}$ ). With a speed-up factor of 110 , and $8 \mathrm{~mm}$ slice thickness, the coronal plane images were acquired approximately at the rate of one frame every 2 seconds. After the motion trials, the table was rotated back to the supine position. Once out of the magnet, the subject performed the bending task again, and one of the investigators (NKM) measured the angle of the right side-bend with a goniometer, positioned between the level of the posterior superior iliac spine and T12 spine, average measures \pm standard deviations (SDs), recorded as $15.6^{\circ} \pm 2.76^{\circ}$ and $14.7^{\circ} \pm 3.43^{\circ}$ for visit 2 and 3 , respectively. The imaging protocols were identically performed for visits 2 and 3 .

\section{Image processing}

Each imaging visit yielded approximately 110 DICOM images for each participant, including those acquired from the motion trials performed. The images represented visual snap-shots in a time series of these bending trials. Fifteen to 20 images from this time series were selected and arranged in a sequence of image files, representing at least two complete sets of bending tasks for each participant, for each visit. Two separate image frame sets were created for each participant for each of the two imaging sessions. These sets of images were then imported into the animation environment and rotoscoped to align and superimpose the individual $3 \mathrm{D}$ vertebral models to their image silhouettes in each image frame. Three-D model registration in these successive image frames yielded the animation of step-wise translational and rotational lumbar spine displacements. The displacements were recorded as two variables: (1) changes in inter-vertebral axes positions (the cranial to the caudal vertebrae) and (2) displacements in individual vertebra (from initial neutral positions, at zero displacement) within the calibrated imaging space. The software created a series of life-like side-ways animation movement and sequentially calculated the step-wise displacements of inter-vertebral axes and individual vertebrae in the coronal plane. For 3D modeling purposes, individualized, motion-segment marionettes were created from axial $\mathrm{T}_{1}$ images for each participant. Image-to-model rotoscoping and registration were performed by a single investigator (NKM) on individual images. Two separate rotoscoping (with displacement quantification) sessions were performed for images acquired and processed from visits 2 and 3 with at least a week between each session. The animation quantified the following variables: (1) inter-vertebral axis translation and rotation (displacements at L2-L3 and L3-L4 junctions), and (2) individual L2, L3, and L4 vertebral displacements (translations and rotations). Following rotoscoping, the variables were quantified as two separate series of datasets; each series representing corresponding data points for the variables from both visits.

\section{Statistical analysis}

Between-session intra-class correlation coefficients (ICCs), coefficients of variation (CV), and Pearson's correlation coefficients were calculated for each measured variable (Table 1). We compared the corresponding data points measured from 2 time series (visit 2 and visit 3 ) to evaluate the reliability of our technique. The CV was calculated, in addition to Pearson's correlation coefficients, with consideration of the intrinsic variability of the speed of motion demonstrated by individual subjects, specifically while going into or coming out of the end-of-range 
positions of the side-bending task. Strengths of the relationship of the between-session measurements were calculated with Pearson's correlation coefficient. All statistical calculations were performed with the statistical software IBM SPSS ver. 21.0 (IBM Corp., Armonk, NY, USA).

\section{Results}

As shown in Table 1, the reliability statistics indicate the quantification technique yielded ICC's ranging from 0.86 to 0.95 for the respective inter-vertebral axis and vertebral translation and rotation measurements. Their CVs ranged between 13\%-17\% (Table 1). Quantifying rotational dis-

Table 1. Reliability statistics for the quantified side-bending variables in the coronal plane

\begin{tabular}{|c|c|c|c|c|c|c|c|c|c|c|}
\hline \multirow[t]{2}{*}{ Between-session comparison } & \multicolumn{2}{|c|}{$\begin{array}{l}\text { Inter-vertebral axis } \\
\text { translation }\end{array}$} & \multicolumn{2}{|c|}{$\begin{array}{l}\text { Inter-vertebral axis } \\
\text { rotation }\end{array}$} & \multicolumn{3}{|c|}{ Vertebral translation } & \multicolumn{3}{|c|}{ Vertebral rotation } \\
\hline & L2-L3 & L3-L4 & L2-L3 & L3-L4 & $\mathrm{L} 2$ & L3 & L4 & L2 & L3 & L4 \\
\hline Intra-class correlation coefficient $(1,2)$ & 0.86 & 0.89 & 0.93 & 0.94 & 0.92 & 0.93 & 0.94 & 0.95 & 0.93 & 0.95 \\
\hline Coefficient of variation (\%) & 16.04 & 17.27 & 14.95 & 14.34 & 14.33 & 13.39 & 13.68 & 13.43 & 14.67 & 13.13 \\
\hline Pearson's correlation coefficient & 0.92 & 0.89 & 0.95 & 0.94 & 0.95 & 0.97 & 0.98 & 0.94 & 0.89 & 0.97 \\
\hline
\end{tabular}
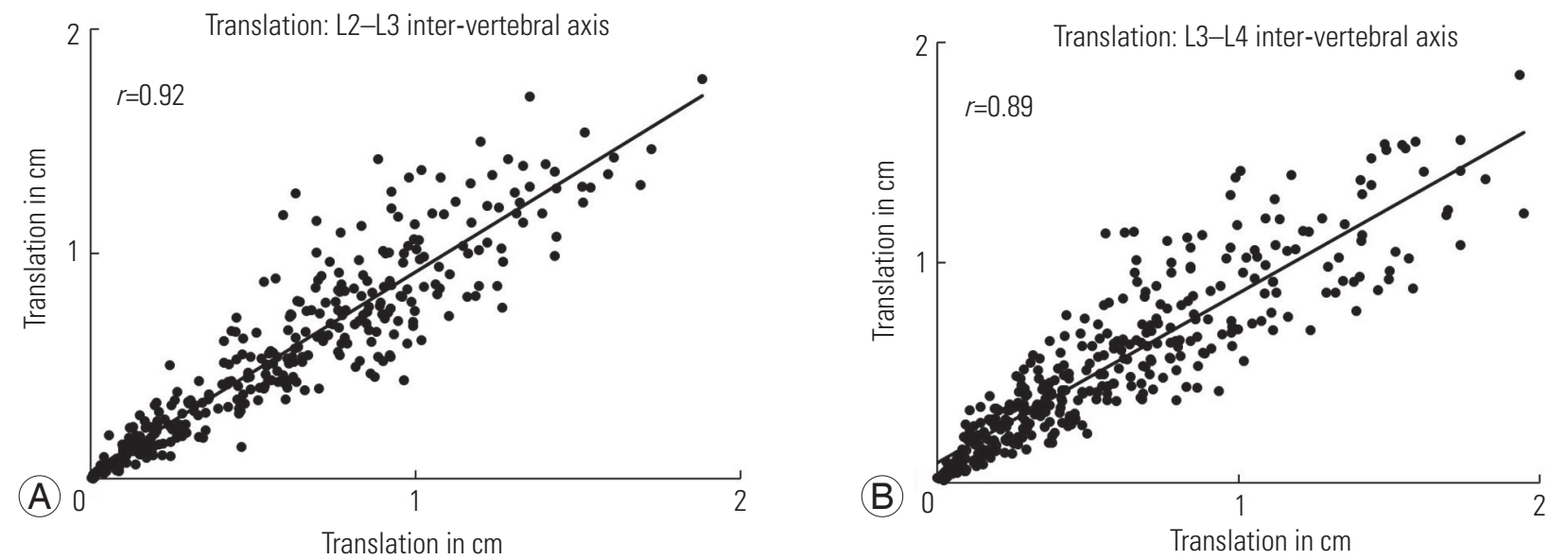

Fig. 2. Strength of relationship between translational displacements quantified at two inter-vertebral axes from two trial sessions. (A) Displacements at the L2-L3 axis. (B) Displacements at the L3-L4 axis.
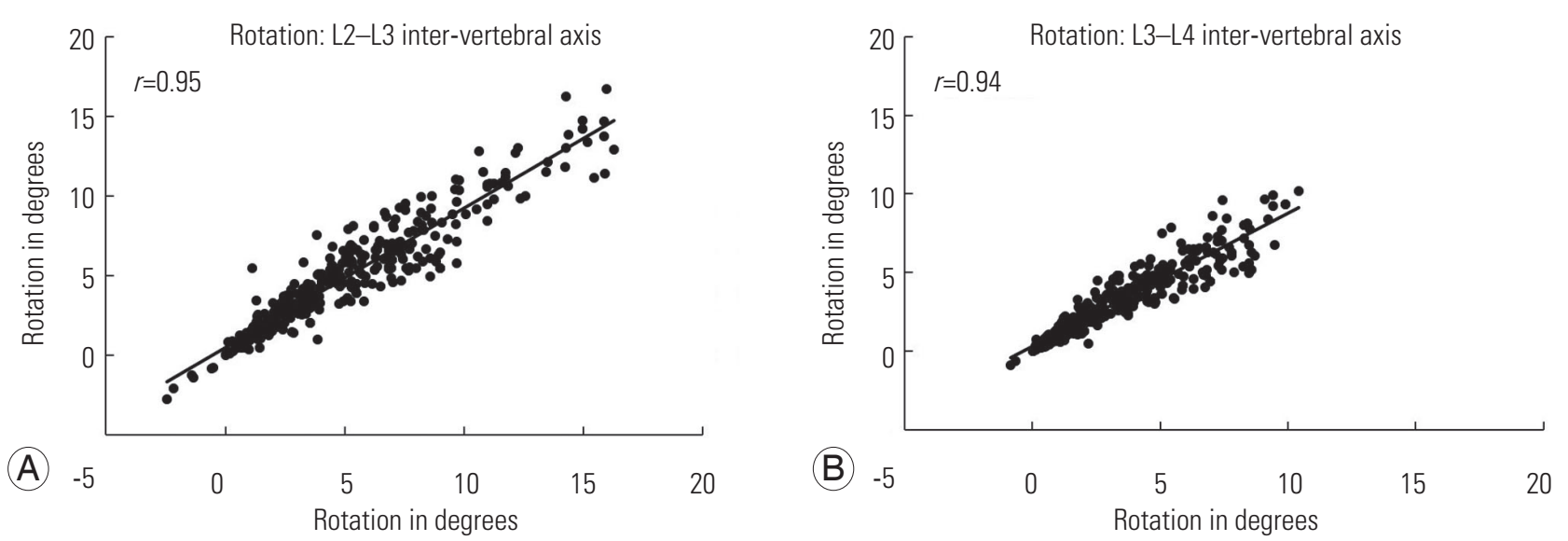

Fig. 3. Strength of relationship between rotational displacements quantified at two inter-vertebral axes from two trial sessions. (A) Displacements at the L2-L3 axis. (B) Displacements at the L3-L4 axis. 
placements demonstrated marginally better reliability results than the translations, both for the inter-vertebral axis and individual vertebral displacements measured (Figs. 2-5). The ICC, CV, and Pearson's correlation coefficients showed marginally better results for the inter-vertebral axis rotational measurements for both the axes in the global reference frame within the animation software. These findings were consistent with our earlier reports in this series. The range of inter-vertebral axes or vertebral displacements, measured from no movement (minimum displacement between two successive frames at the neutral or at the end-of-range position) to maximum, was as follows: inter-vertebral axis translation $(2.35 \mathrm{~cm} / \mathrm{L} 2-$ L3), inter-vertebral axis rotation (17.29\%/L2-L3), vertebral translation $(1.34 \mathrm{~cm} / \mathrm{L} 2)$, and vertebral rotation $\left(15.86^{\circ}\right.$ / L2) displacements; inter-vertebral axis translation (1.95 $\mathrm{cm} / \mathrm{L} 3-\mathrm{L} 4)$, inter-vertebral axis rotation $\left(11.42^{\circ} / \mathrm{L} 3-\mathrm{L} 4\right)$, vertebral translation $(1.23 \mathrm{~cm} / \mathrm{L} 3)$, and vertebral rotation $\left(13.09^{\circ} / \mathrm{L} 3\right)$; and vertebral translation $(0.94 \mathrm{~cm} / \mathrm{L} 4)$ and vertebral rotation $\left(8.49^{\circ} / \mathrm{L} 3\right)$; the maximum range of movements was observed at the upper axis (L2-L3) and the cranial L2 vertebra for all translation and rotations. The range of excursions, quantified at the upper inter- vertebral axis and the L2 vertebra, were similar to overall range of motion reported in earlier studies studying physiologic range of the lumbar spine motion [14,20,21].

\section{Discussion}

The new imaging, image processing, and quantification approach presented in this study yields proof-of-concept results indicating fast, dynamic, weight-bearing MR imaging of the lumbar spine could be performed in human subjects, and lumbar spine motion could be quantified using an animation-based approach. Some of current approaches used to detect micro-motion in implanted joint components require pre-operative placement of microbeads in the peri-articular bone. These techniques are expensive, and their routine use to detect hyper-mobile joint segments is also restricted by the associated risks of ionizing radiation. Our earlier results, testing the accuracy of these real-time MR sequences for mapping inter-vertebral displacements of translation and rotation end-point displacements in pig models, have been encouraging. The reliability results from the present study suggest that, with further validation of this technique's accuracy, our
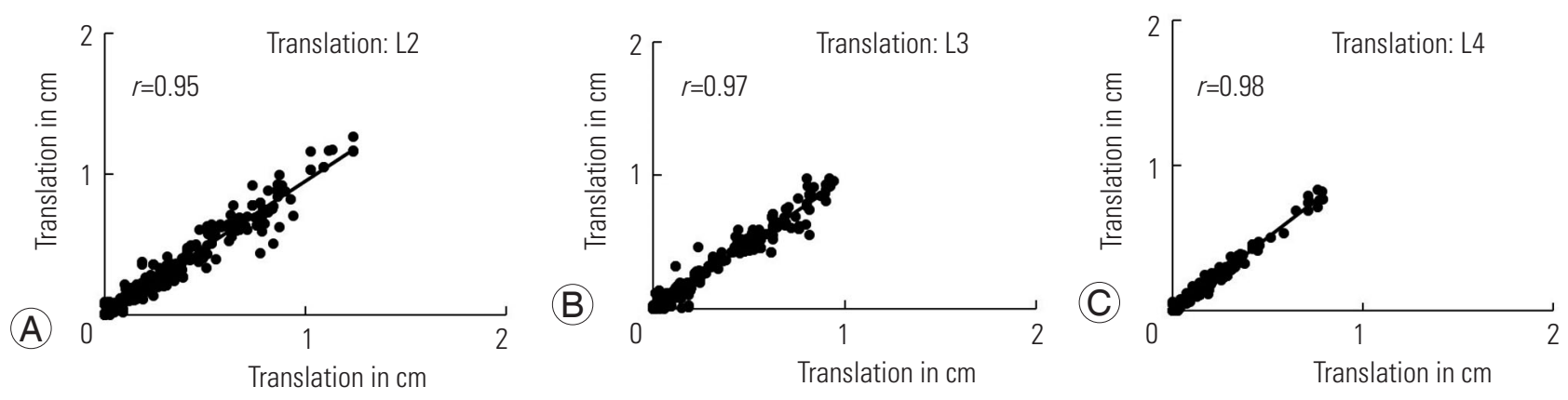

Fig. 4. Strength of relationship between translational displacements quantified for individual lumbar vertebrae from two trial sessions. (A) Displacements in L2. (B) Displacements in L3. (C) Displacements in L4.
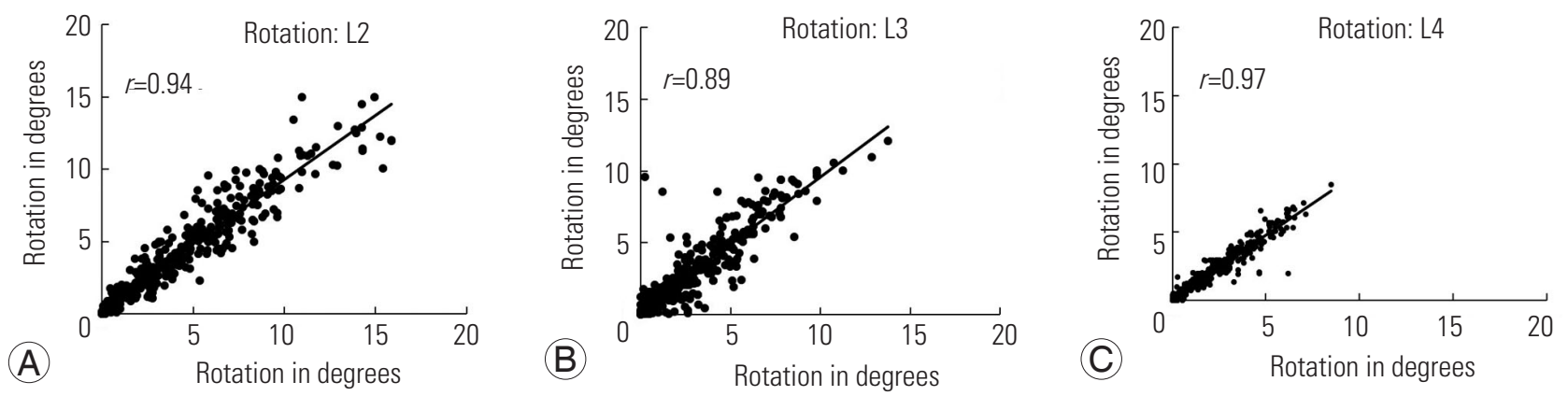

Fig. 5. Strength of relationship between rotational displacements quantified for individual lumbar vertebrae from two trial sessions. (A) Displacements in L2. (B) Displacements in L3. (C) Displacements in L4. 
MR imaging protocol could be used to compare betweensession displacements in inter-vertebral motion and intervertebral disc deformation in the lumbar spine. Without definite, established, clinical cut-offs to diagnose dynamic lumbar inter-vertebral instability, and in absence of dynamic MRI-based evaluation techniques, our protocol can be used to detect inter-vertebral motion through a given range of excursion.

The reliability results, expressed as the $\mathrm{CV}$ percentages, demonstrate apparently larger variances (when compared to the correlation analysis) in mapping between-session quantification. The relatively greater CV\% values calculated in our study may have occurred because of certain limitations of the acquisition pace with the dynamic MRI sequences. Since calculating the CVs involves the SDs of each measured displacement, the between-trial and between-session variations in the CV\% may have resulted from differences in the individual participant's speed of movement at sections within the range of motion set for the trials (between-trial and between-session). Accordingly, the limitation of the current dynamic MRI sequences of image acquisition may have yielded varied images where the subjects moved inconsistently through the range, possibly resulting in the greater data point dispersion at these stretches of motion. No CV were calculated for these variances since the subjects learned the timed motion before the actual scanning sessions and demonstrated little variability in completing the entire bending protocol.There are a few limitations of this work that should be noted. First, the image processing and 3D model registration, performed manually in this study, are time-consuming (Fig. 6). Fast, automated, pixel-based techniques for model registration were not used in this study because of a technical limitation of the animation software. Second, given the limitation of current MR imaging technology, the speed (frame rate) of dynamic image acquisition were not as fast as fluoroscopy-based quantification techniques. Though our open-MRI system allows static weight-bearing image acquisition, lumbar movement is restricted in the sagittal plane because of the magnet bore shape. Thus, our study used a side-bending protocol in the coronal plane. Acquiring images in the sagittal plane (flexion-extension) would have been ideal since it allows for a greater range of motion and visualization of useful anatomy for model superimposition to detect lumbar instability $[20,22,23]$. However, the range of inter-vertebral axis motion quantified in our study matches with earlier

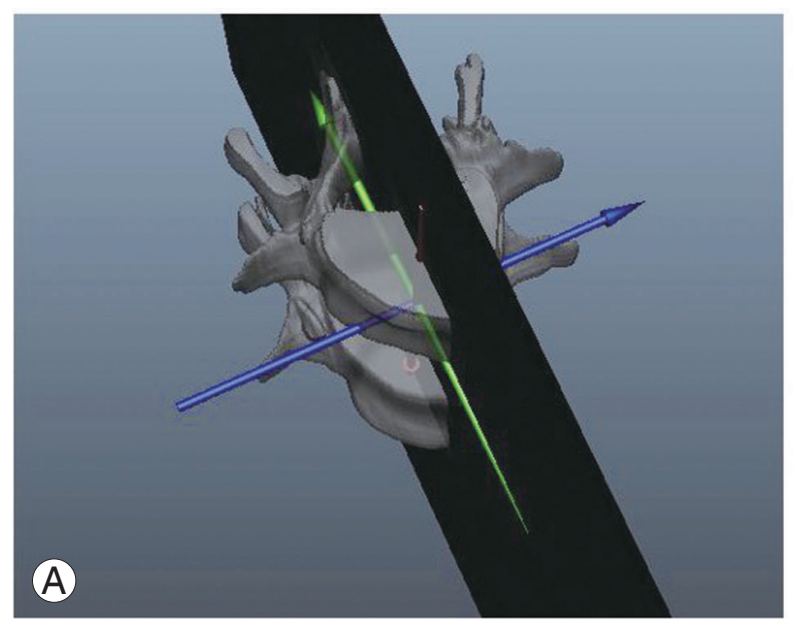

Vertebrae registered to a sagittal image frame

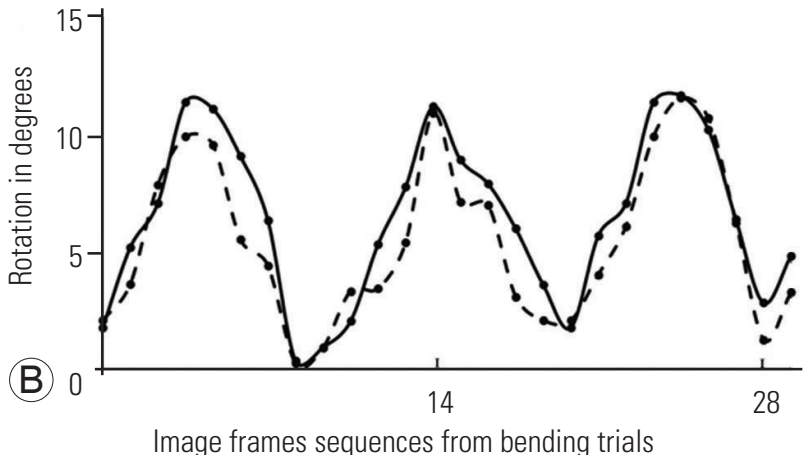

Fig. 6. Representational figures. (A) Figure showing a couple of vertebrae registered to a sagittal image frame in the virtual environment. Vertebral models in this study were registered to coronal images, in a sequence [16]. (B) Graph showing data points from a 3-cycle right side-bending trial for the $\mathrm{L} 2-\mathrm{L} 3$ inter-vertebral axis rotation. The baseline is the neutral upright position with the ascent denoting right sidebend followed by a return to the upright. The two imaging study visits, visit 2 and 3 are represented by the continuous and the broken lines respectively.

reports and may help develop similar MRI-based realtime motion assessment involving automated algorithms, for faster and accurate quantification [24-26]. Testing this technique's accuracy with dynamic spine displacements, either comparing outcomes to another established spine motion tracking technique, or developing an internal accuracy measurement system, will be the next step in the process.

Developing high-definition, faster, volume-based (voxel) MR image acquisition systems, and the availability of pixel-based algorithms for image-quality detection and accurate 3D model registration techniques, will allow $\mathrm{MR}$ imaging for dynamic assessment of spine motion to be available for future clinical use. Furthermore, developing faster real-time imaging sequences, voxel-based imag- 
ing, and automated 3D model rendition techniques may enhance the usage of MR-only approaches for quantifying inter-vertebral displacement in all degrees-of-freedom in the global co-ordinate system. In the technique presented in this study, improving dynamic image resolution and calibrating the imaging volume, as well as refining the accuracy of 3D model alignment over the uni-planar image slices, can help increase the quality of this technique in quantifying complex joint motion [24,27-31].

\section{Conclusions}

This experimental technique may be developed to enhance speed of image acquisition and accuracy of quantification to be used for diagnostic applications. This technique can potentially be used to study in-vivo spine stability, and to assess outcomes of surgical and non-surgical interventions applied to manage pathological spine motion.

\section{Conflict of Interest}

No potential conflict of interest relevant to this article was reported.

\section{References}

1. Vaisy M, Gizzi L, Petzke F, Consmuller T, Pfingsten M, Falla D. Measurement of lumbar spine functional movement in low back pain. Clin J Pain 2015;31:87685.

2. Mellor FE, Muggleton JM, Bagust J, Mason W, Thomas PW, Breen AC. Midlumbar lateral flexion stability measured in healthy volunteers by in vivo fluoroscopy. Spine 2009;34:E811-7.

3. Mellor FE, Thomas PW, Thompson P, Breen AC. Proportional lumbar spine inter-vertebral motion patterns: a comparison of patients with chronic, non-specific low back pain and healthy controls. Eur Spine J 2014;23:2059-67.

4. Papi E, Koh WS, McGregor AH. Wearable technology for spine movement assessment: a systematic review. J Biomech 2017;64:186-97.

5. Chiari L, Della Croce U, Leardini A, Cappozzo A. Human movement analysis using stereophotogrammetry: part 2: instrumental errors. Gait Posture 2005;21:197-211.

6. Reutlinger C, Hasler C, Scheffler K, Buchler P. Intra- operative determination of the load-displacement behavior of scoliotic spinal motion segments: preliminary clinical results. Eur Spine J 2012;21:S860-7.

7. Leardini A, Chiari L, Della Croce U, Cappozzo A. Human movement analysis using stereophotogrammetry: part 3: soft tissue artifact assessment and compensation. Gait Posture 2005;21:212-25.

8. Ha KY, Son JM, Im JH, Oh IS. Risk factors for adjacent segment degeneration after surgical correction of degenerative lumbar scoliosis. Indian J Orthop 2013;47:346-51.

9. Ha TH, Saber-Sheikh K, Moore AP, Jones MP. Measurement of lumbar spine range of movement and coupled motion using inertial sensors: a protocol validity study. Man Ther 2013;18:87-91.

10. Meakin JR, Gregory JS, Aspden RM, Smith FW, Gilbert FJ. The intrinsic shape of the human lumbar spine in the supine, standing and sitting postures: characterization using an active shape model. J Anat 2009;215:206-11.

11. Meakin JR, Smith FW, Gilbert FJ, Aspden RM. The effect of axial load on the sagittal plane curvature of the upright human spine in vivo. J Biomech 2008;41:2850-4.

12. Meakin JR, Gregory JS, Smith FW, Gilbert FJ, Aspden RM. Characterizing the shape of the lumbar spine using an active shape model: reliability and precision of the method. Spine 2008;33:807-13.

13. Mahato NK, Sybert D, Law T, Clark B. Effects of spine loading in a patient with post-decompression lumbar disc herniation: observations using an open weight-bearing MRI. Eur Spine J 2017;26:17-23.

14. Shin JH, Wang S, Yao Q, Wood KB, Li G. Investigation of coupled bending of the lumbar spine during dynamic axial rotation of the body. Eur Spine J 2013;22:2671-7.

15. Tan Y, Aghdasi BG, Montgomery SR, Inoue H, Lu C, Wang JC. Kinetic magnetic resonance imaging analysis of lumbar segmental mobility in patients without significant spondylosis. Eur Spine J 2012;21:2673-9.

16. Mahato NK, Montuelle S, Goubeaux C, et al. Quantification of intervertebral displacement with a novel MRI-based modeling technique: assessing measurement bias and reliability with a porcine spine model. Magn Reson Imaging 2017;38:77-86.

17. Mahato NK, Montuelle S, Cotton J, Williams S, Thomas J, Clark B. Development of a morphology- 
based modeling technique for tracking solid-body displacements: examining the reliability of a potential MRI-only approach for joint kinematics assessment. BMC Med Imaging 2016;16:38.

18. Gatesy SM, Baier DB, Jenkins FA, Dial KP. Scientific rotoscoping: a morphology-based method of 3-D motion analysis and visualization. J Exp Zool A Ecol Genet Physiol 2010;313:244-61.

19. Brainerd EL, Baier DB, Gatesy SM, et al. X-ray reconstruction of moving morphology (XROMM): precision, accuracy and applications in comparative biomechanics research. J Exp Zool A Ecol Genet Physiol 2010;313:262-79.

20. Dvorak J, Panjabi MM, Chang DG, Theiler R, Grob D. Functional radiographic diagnosis of the lumbar spine: flexion-extension and lateral bending. Spine 1991;16:562-71.

21. Yao Q, Wang S, Shin JH, Li G, Wood K. Motion characteristics of the lumbar spinous processes with degenerative disc disease and degenerative spondylolisthesis. Eur Spine J 2013;22:2702-9.

22. Dvorak J, Panjabi MM, Novotny JE, Chang DG, Grob D. Clinical validation of functional flexionextension roentgenograms of the lumbar spine. Spine 1991;16:943-50.

23. Weishaupt D, Schmid MR, Zanetti M, et al. Positional MR imaging of the lumbar spine: does it demonstrate nerve root compromise not visible at conventional MR imaging? Radiology 2000;215:247-53.

24. Martin DE, Greco NJ, Klatt BA, Wright VJ, Anderst WJ, Tashman S. Model-based tracking of the hip: implications for novel analyses of hip pathology. J
Arthroplasty 2011;26:88-97.

25. Zhu Z, Massimini DF, Wang G, Warner JJ, Li G. The accuracy and repeatability of an automatic 2D-3D fluoroscopic image-model registration technique for determining shoulder joint kinematics. Med Eng Phys 2012;34:1303-9.

26. Borotikar BS, Sipprell WH 3rd, Wible EE, Sheehan FT. A methodology to accurately quantify patellofemoral cartilage contact kinematics by combining $3 \mathrm{D}$ image shape registration and cine-PC MRI velocity data. J Biomech 2012;45:1117-22.

27. Hasegewa K, Kitahara K, Hara T, Takano K, Shimoda $\mathrm{H}$. Biomechanical evaluation of segmental instability in degenerative lumbar spondylolisthesis. Eur Spine J 2009;18:465-70.

28. Lurie JD, Doman DM, Spratt KF, Tosteson AN, Weinstein JN. Magnetic resonance imaging interpretation in patients with symptomatic lumbar spine disc herniations: comparison of clinician and radiologist readings. Spine 2009;34:701-5.

29. Lurie JD, Moses RA, Tosteson AN, et al. Magnetic resonance imaging predictors of surgical outcome in patients with lumbar intervertebral disc herniation. Spine 2013;38:1216-25.

30. Pearson AM, Spratt KF, Genuario J, et al. Precision of lumbar intervertebral measurements: does a computer-assisted technique improve reliability? Spine 2011;36:572-80.

31. Zhao K, Yang C, Zhao C, An KN. Assessment of noninvasive intervertebral motion measurements in the lumbar spine. J Biomech 2005;38:1943-6. 